\title{
Tuberkulose 2019 - eine Herausforderung auch für die Radiologie
}

\section{Tuberculosis 2019 - a Challenge also for Radiology}

\section{Einleitung}

Die Inzidenz der Tuberkulose in Mittel- und Südeuropa hat in den letzten Jahren aufgrund erhöhter Migrationsbewegungen deutlich zugenommen (Pontarelli A et al. Travel Med Infect Dis 2019; 27: 29 -45). Die Primärtuberkulose hat ihren Ursprung in einem Primärherd im Lungenparenchym, von welchem die Ausbreitung zentripetal lymphogen erfolgt und letztlich zur typischen hilären Lymphadenitis führt. In konventionellen Röntgenaufnahmen des Thorax zeigt sich der frische Primärherd oft als kleinfleckiges Infiltrat in der Lungenperipherie mit ipsilateraler hilärer Lymphadenopathie und ggf. begleitendem Pleuraerguss.
Die Postprimärtuberkulose ist bedingt durch eine Reaktivierung von Tuberkelbakterien und kann diverse Organsysteme betreffen. Pulmonal zeigen sich häufig solitäre tuberkulöse Herde im Apex oder eine miliare Form mit oberlappenbetonten feinen Noduli. Die häufigste extrapulmonale Manifestation betrifft wiederum die Lymphknoten. Somit ist neben der Röntgen- und Ultraschalldiagnostik ggf. auch eine Computertomografie (CT) des Thorax indiziert, da die Sensitivität zum Nachweis von hilären und mediastinalen Lymphknotenvergrößerungen deutlich höher ist. Ein abdomineller Befall ist seltener, erfordert jedoch eine erweiterte Diagnostik.
Die folgenden Fälle stellen eine typische und eine seltene Form der Postprimärtuberkulose bei einem pädiatrischen und einem erwachsenen Patienten dar.

\section{Fallbeschreibung 1}

Eine 12-jährige Patientin stellte sich mit seit einigen Monaten bestehender schmerzhafter Schwellung links axillär sowie infraklavikulär in der Ambulanz der Kinderklinik vor. Das Mädchen war ein halbes Jahr zuvor aus Somalia nach Deutschland eingereist. Seitdem hatte das Kind ca. $10 \mathrm{~kg}$ an Gewicht verloren. Intermittierend sei Fieber sowie trockener Husten aufgetreten. Die Eltern
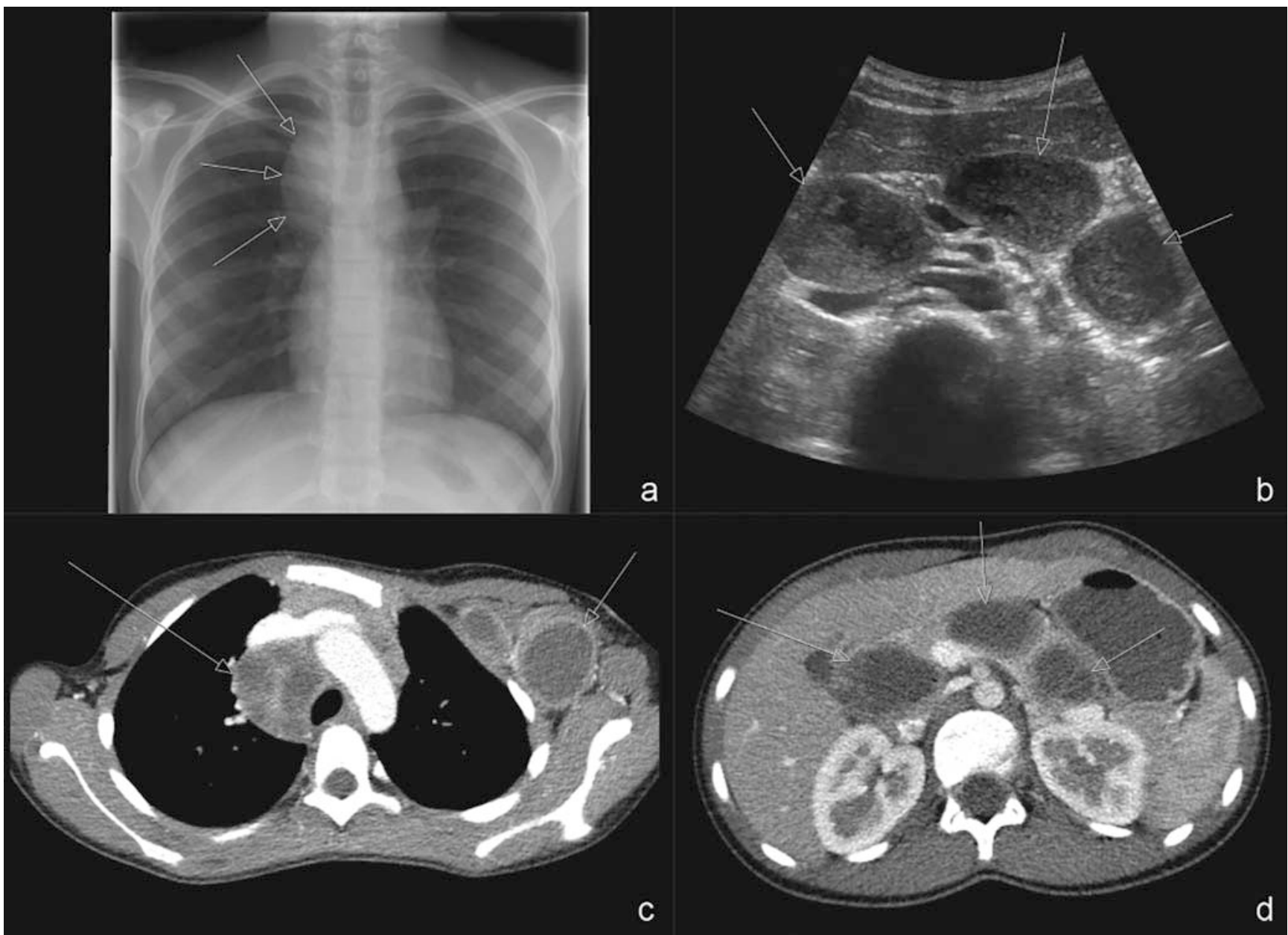

- Abb. 1 Konventionelle Röntgenaufnahme des Thorax mit Nachweis einer Verbreiterung des oberen Mediastinal-Schattens rechtsseitig a. Sonografie des Abdomens mit mehreren pathologisch vergrößerten mesenterialen Lymphknoten b. Korrespondierende kontrastgestützte CT mit Nachweis vergrößerter, zentral hypodenser mediastinaler und links axillärer c sowie mesenterialer d Lymphknoten mit randständiger Kontrastanreicherung. 


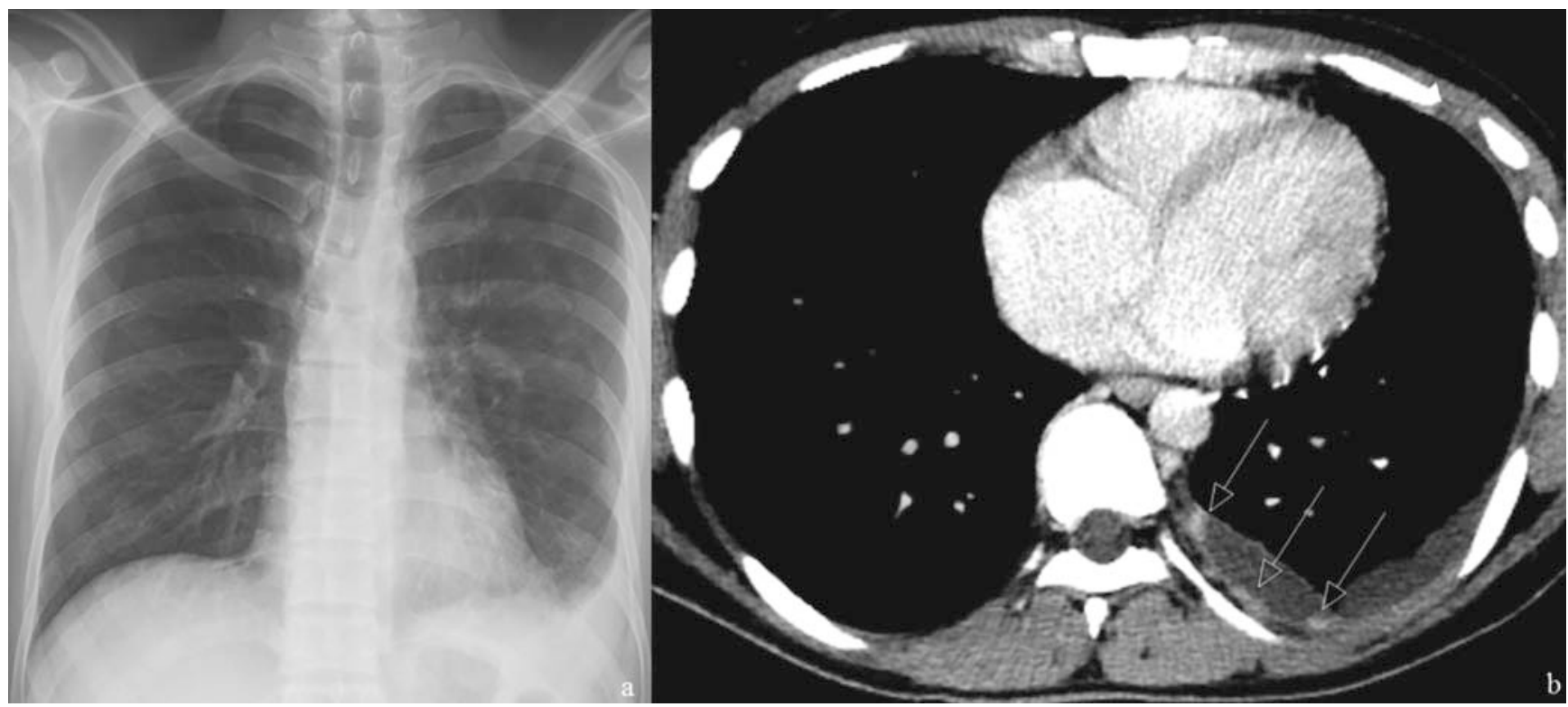

- Abb.2 Die konventionelle Röntgenaufnahme des Thorax in p. a.-Strahlengang a zeigt bis auf einen Pleuraerguss links keine Auffälligkeiten. In der CT-Untersuchung in axialer Schnittführung b ist eine atypische Konfiguration des Pleuraergusses mit nodulären Kontrastmittelanreicherungen der Pleura (Pfeile) erkennbar. Auf der Gegenseite ist kein Pleuraerguss nachweisbar.

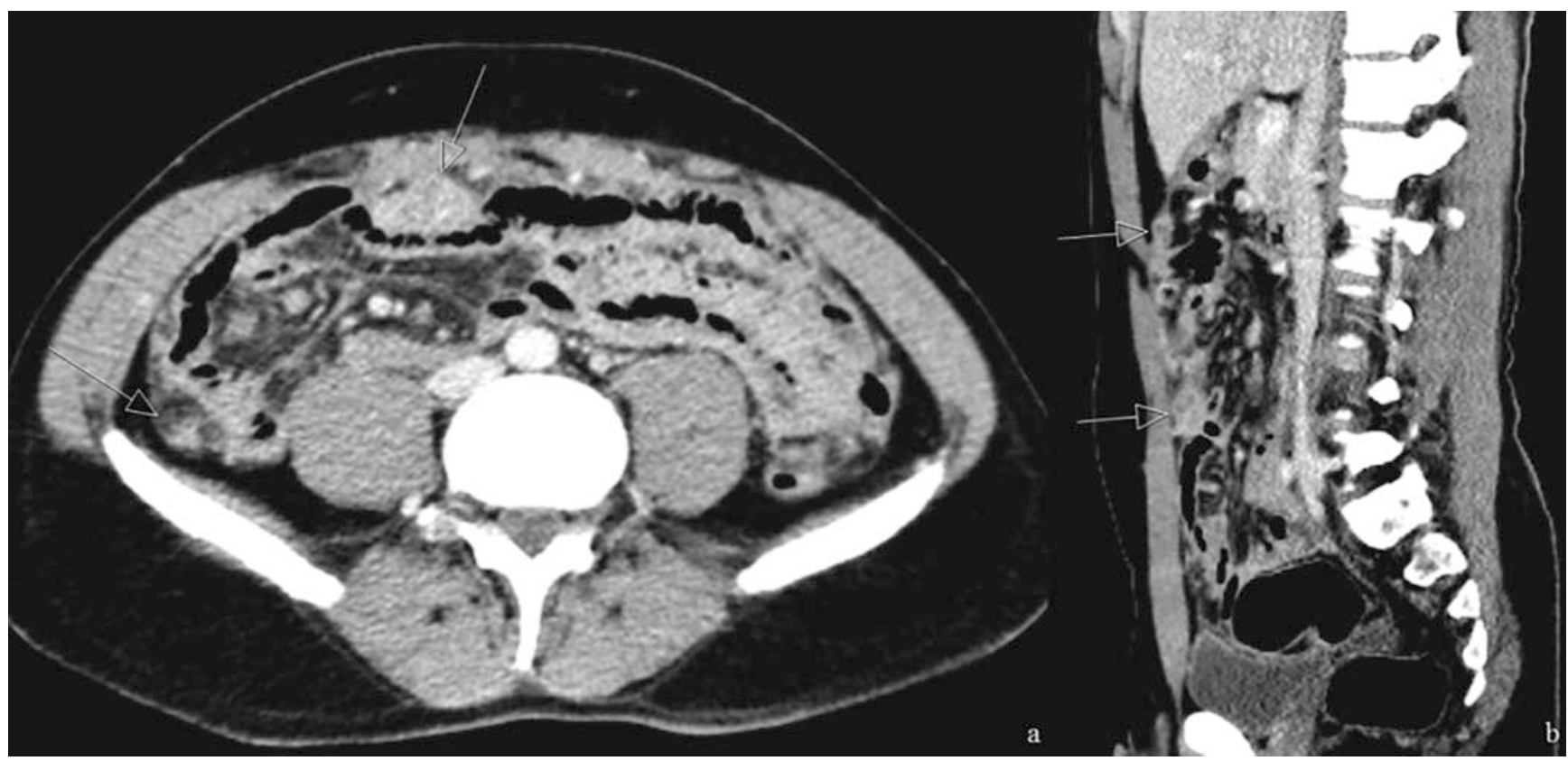

- Abb. 3 Axiale a und sagittale b multiplanare Rekonstruktion der CT-Untersuchung des Abdomens mit knotigen peritonealen Veränderungen (Pfeile), auffälligen mesenterialen Lymphknoten und diffuser Imbibierung des mesenterialen Fettgewebes.

berichteten, dass das Mädchen bereits im Kleinkindalter an Tuberkulose erkrankt war. Die klinische Untersuchung bestätigte die vermutete linksaxilläre Lymphadenopathie, auch abdominell waren Resistenzen palpabel.

In der initialen Röntgen-Thorax-Untersuchung fiel eine rechtsbetonte Verbreite- rung des oberen Mediastinal-Schattens auf. Sonografisch waren pathologisch vergrößerte mesenteriale Lymphknoten nachweisbar ( $\mathbf{A b b}$. 1). In Zusammenschau der Befunde bestand der Verdacht auf eine Lymphknotentuberkulose, differenzialdiagnostisch wurde ein Lymphom in Betracht gezogen. Eine CT des Thorax sowie des miterfassten Oberbauches bestätigte die mediastinale, axilläre und mesenteriale Lymphadenopathie ( $\triangleright$ Abb. 1). Der Quantiferon-Test war positiv und auch histologisch konnte die Infektion in einem exstirpierten infraklavikulären Lymphknoten bestätigt werden. 
Eine spezifische antibiotische Therapie wurde eingeleitet. Nach komplikationslosem Verlauf konnte diese nach 6 Monaten beendet werden.

\section{Fallbeschreibung 2}

Ein 20-jähriger Mann aus Somalia wurde aufgrund seit 1 Monat bestehender linksseitiger Thoraxschmerzen sowie akut stärkster Bauchschmerzen mit Abwehrspannung in die Notaufnahme gebracht. Seit 1 Woche bestand zusätzlich Fieber. Laborchemisch war eine deutliche Erhöhung des CRP auf $233 \mathrm{mg} / \mathrm{l}$ (Normwert: $<5 \mathrm{mg} / \mathrm{l}$ ) und eine Anämie erkennbar.

Die Röntgenaufnahme des Thorax zeigte einen linksseitigen Pleuraerguss ( $\triangleright$ Abb.2), die Sonografie des Abdomens geringe Mengen freier Flüssigkeit und eine Hepatomegalie.

Aufgrund der Klinik und Laborkonstellation wurde eine CT des Thorax und Abdomens durchgeführt. Diese zeigte eine Kontrastanreicherung der Pleura mit gefangenem Ergussanteil im Lappenspalt ( $\triangleright$ Abb. 2). Abdominell waren vergrößerte mesenteriale Lymphknoten, peritoneale Knoten, eine peritoneale Kontrastanreicherung, mesenteriale Fettgewebs-Imbibierungen sowie geringe Mengen freier Flüssigkeit nachweisbar ( $\mathbf{A b b}$. 3). Aufgrund der Gesamtkonstellation wurde der Verdacht auf eine tuberkulöse Peritonitis und Pleuritis gestellt, differenzialdiagnostisch wurde auch eine Peritoneal-/Pleurakarzinose in Betracht gezogen.

Die Tuberkulose wurde durch einen positiven Quantiferon-Test bestätigt. Nach einer laparoskopischen Probenentnahme konnten in einem der peritonealen Knoten Mykobakterien nachgewiesen werden. Eine spezifische antibiotische Therapie wurde eingeleitet und der Patient konnte bei unkompliziertem Verlauf nach 4 Wochen entlassen werden.

\section{Diskussion}

Die Primärtuberkulose verläuft oft subklinisch. Sie ist jedoch die häufigste aktive Form bei jüngeren Kindern, während Jugendliche meist von der postprimären Form betroffen sind (Cruz AT et al. Pediatr Infect Dis J 2013; 32: 937 - 941). Fall 1 dokumentiert eine häufige Form der Postprimärtuberkulose mit Lymphknotenbefall, Fall 2 eine seltenere Manifestation mit pleuralem und peritonealem Befall. Betroffene Lymphknoten stellen sich CT-morphologisch typischerweise zentral hypodens mit randständiger Kontrastanreicherung dar (vgl. Fall 1). Dies ist bedingt durch zentrale verkäsende Nekrosen mit peripheren granulomatös-entzündlichen Gewebeveränderungen (Nachiappan AC et al. Radiographics 2017; 37: 52 - 72).

Bei abdominellem Befall kommt der Schnittbilddiagnostik eine entscheidende Rolle zu (Lee WK et al. Clin Radiol 2012; 67: 596 - 604). Der tuberkulösen Peritonitis wie in Fall 2 liegt pathophysiologisch meist eine Ruptur eines befallenen mesenterialen Lymphknotens oder eine lymphogene mikro-noduläre Aussaat zugrunde (Pereira JM et al. Eur J Radiol 2005; 55: 173 - 180). Aufgrund der Heterogenität der Befalls-Muster sind jedoch die Differenzialdiagnosen weitreichend und schließen auch grundsätzlich maligne Erkrankungen mit ein.

\section{Schlussfolgerung}

Aufgrund der zunehmenden Inzidenz von Tuberkulose auch in den Industriestaaten ist die Kenntnis charakteristischer Erscheinungsbilder der Infektion für den Radiolo- gen essenziell. Bei Tuberkuloseverdacht muss eine entsprechende Abklärung (ggf. inklusive histologischer Sicherung) empfohlen werden, um den Patienten adäquat zu therapieren und eine weitere Ausbreitung zu verhindern.

Interessenkonflikt

Die Autoren geben an, dass kein Interessenkonflikt besteht.

\section{Autorinnen/Autoren}

André Lollert ${ }^{1}$, Gundula Staatz ${ }^{1}$, Florian Jungmann²

\footnotetext{
1 Department of Diagnostic and Interventional Radiology, Section of Pediatric Radiology, Medical Center of the Johannes-GutenbergUniversity Mainz, Germany

${ }^{2}$ Department of Diagnostic and Interventional Radiology, Medical Center of the JohannesGutenberg-University Mainz, Germany
}

\section{Korrespondenzadresse}

\author{
Dr. med. André Lollert \\ Department of Diagnostic and \\ Interventional Radiology \\ Section of Pediatric Radiology \\ Medical Center of the Johannes-Gutenberg- \\ University \\ Langenbeckstr. 1 \\ 55131 Mainz \\ Tel.: ++49/6131/172445 \\ Fax: ++49/6131/17 6682 \\ andre.lollert@unimedizin-mainz.de
}

\section{Bibliografie}

DOI https://doi.org/10.1055/a-0853-2732

Online-Publikation: 2019

Fortschr Röntgenstr 2019; 191: 354-356

(c) Georg Thieme Verlag KG, Stuttgart · New York ISSN 1438-9029 